\title{
ANESTRO POSPARTO EN GANADO BOVINO EN EL TRÓPICO
}

\author{
POSTPARTUM ANESTRUS I N CATTLE IN THE TROPIC
}

\begin{abstract}
Giovanni Báez S*, M.Sc, Henry Grajales L, Ph.D.
Universidad Nacional de Colombia, Facultad de Medicina Veterinaria y de Zootecnia, Departamento de Ciencias para la Producción Animal, Grupo de investigación en Fisiología de la Reproducción. Bogotá, Colombia. *Correspondencia: gmbaezs@unal.edu.co
\end{abstract}

Recibido: Noviembre 7 de 2008; Aceptado: Agosto 30 de 2009

\section{RESUMEN}

La productividad y rentabilidad de las empresas ganaderas depende en gran medida de la capacidad fisiológica de las hembras para cumplir con el objetivo de tener una cría por año. Un factor determinante para lograrlo es reducir al máximo el periodo de anestro posparto, que es el tiempo luego del parto durante el cual no existen suficientes niveles pulsátiles de hormona luteinizante (LH) que permitan un desarrollo final del folículo y la consiguiente ovulación. Dos factores intervienen principalmente en este fenómeno: la nutrición y el amamantamiento. Existen señales metabólicas que indican al eje hipotálamo-hipófisis que se ha alcanzado un equilibrio en el balance energético para que este inicie la secreción de hormona liberadora de gonadotropinas $(\mathrm{GnRH})$ y se inicie el restablecimiento de la actividad reproductiva. Por su parte, el estimulo que ejerce el ternero sobre la vaca genera secreción de endorfinas endógenas a nivel hipotalámico que también van a inhibir la liberación de $\mathrm{GnRH}$. Otros factores que modulan los efectos de la nutrición y el amamantamiento son la edad, numero de partos, raza, estrés calórico y factores sociales (efecto macho y hembra). Generalmente, luego de la primera ovulación, se presenta un ciclo estral de corta duración que corresponde a una luteolisis temprana en respuesta a la prostaglandina F2 alfa (PGF2 $\alpha$ ) de origen uterino; sin embargo, los niveles de progesterona producidos durante este corto periodo ejercen un importante papel en el establecimiento de la ciclicidad subsecuente.

Palabras clave: Nutrición, amamantamiento, crecimiento folicular, ovulación, ciclicidad.

\section{ABSTRACT}

Both productivity and profitability in cattle operations are depending on the physiologic capacity of the cow to obtain one calf per year. The most important fact for achieving this goal is reducing the postpartum period, when pulses of luteinizing hormone (LH) are not enough to allow a final development and ovulation of dominant follicles. Two main factors 
affect this phenomenon: nutrition and suckling. Several metabolic signals act over the hypotalamus-hypophysis axis indicating the energy balance and allowing the onset of gonadotropin releasing hormone $(\mathrm{GnRH})$ secretion and the onset of cycling. On the other hand, the calf generates endogenous endorphins secretion and inhibition of GnRH release. Further modulators of nutrition and suckling effects are age, parity, breed, heat stress and social factors (male and female effect). Generally, after the first ovulation, a short estrous cycle is shown, probably because of an early luteolisis in response to prostaglandin F2 alpha (PGF2 $\alpha$ ) release by the uterus; however, progesterone released by the short estrous cycle corpus luteum plays an important role on the establishment of the subsequent cyclicity.

Key words: Nutrition, suckling, folicullar growth, ovulation, ciclicity

\section{NTRODUCCI ÓN}

La ganadería en el trópico se caracteriza por presentar una baja producción por animal, así como por fluctuaciones entre épocas, ya que durante la época de lluvias los animales se encuentran relativamente bien alimentados, produciendo a su máxima capacidad; pero en la época seca la alimentación es pobre, reduciendo notablemente la producción (1).

La productividad ganadera en términos de carne y leche es dependiente del desempeño reproductivo, y este a su vez se encuentra íntimamente relacionado con la fertilidad. De esta manera un intervalo entre partos normal incrementa tanto el número de terneros nacidos como la cantidad de leche producida por vaca durante la vida útil.

El objetivo general de esta revisión fue compilar algunos aspectos determinantes del período fisiológico de posparto, haciendo énfasis en los factores que lo afectan particularmente en las regiones tropicales.

\section{FI SI OLOGÍ A DEL POSPARTO}

El anestro postparto se puede definir como el período después del parto durante el cual las vacas no muestran señales conductuales de estro (2). La primera ovulación posparto se presenta cuando existe un folículo dominante y pulsos de LH (Hormona Luteinizante) cada hora (3). Durante el posparto temprano los pulsos de LH son secretados cada 3 a 6 h (4).

Los principales factores que afectan la duración del anestro postparto son el estado nutricional (condición corporal) y el amamantamiento. Algunos otros factores como la raza, edad, número de partos, producción de leche, temporada de parto, presencia del toro, involución uterina, distocias y estado de salud general modulan los efectos provocados por estos dos factores principales (5).

\section{NUTRI CI ÓN}

Balance energético. El balance energético negativo reduce la disponibilidad de glucosa e incrementa la movilización de reservas corporales. El metabolismo basal, la actividad, el crecimiento y la lactancia tienen prioridad sobre los procesos reproductivos, como el reinicio de la ciclicidad y el establecimiento y mantenimiento de una nueva preñez (6)

Un inadecuado consumo de nutrientes en relación con las demandas metabólicas es un factor que contribuye a prolongar el anestro posparto y aunque la naturaleza de estos factores y sus interacciones es compleja y poco conocida, muchos de ellos parecen actuar por vía de mecanismos hormonales (7). La transición de un balance energético negativo a uno positivo durante la lactancia está asociada al incremento en la frecuencia de pulsos de LH (8), lo cual sugiere que la secreción pulsátil de LH puede ser inhibida hasta tanto no se alcance el nadir del balance energético.

En vacas de leche se ha relacionado el intervalo entre partos y la primera ovulación con el estatus metabólico (9), encontrando 
una relación inversamente proporcional entre balance energético y la reanudación de actividad ovárica. Canfield y Butler (8), encontraron que la primera ovulación ocurrió aproximadamente a los 14 días después del máximo balance energético negativo. En ganado criollo se reportó el nadir entre los días 50-60 posparto (10). Galina y Arthur (11) reportan para vacas Bos indicus en condiciones normales de explotación valores mínimos entre los 90 y 120 días posparto y una ligera recuperación hasta los 180 días.

En regiones tropicales, vacas que paren con condición corporal aceptable y que mantienen esta condición durante el posparto, tienen mayores tasas reproductivas que vacas con baja condición (11).

Hormonas metabólicas. En los primeros estudios relacionados con la interacción nutrición-reproducción (6), se establecía que las concentraciones de glucosa en sangre relacionan el estado nutricional con la función reproductiva en el hipotálamo. Vacas con concentración de glucosa reducida disminuyen su cantidad de progesterona en plasma (12). Aunque las concentraciones de glucosa en bovinos son muy constantes en comparación con monogástricos, la insulina regula la utilización de glucosa por parte de las células. La insulina estimula la liberación de GnRH de fragmentos hipotalámicos in vitro, cuando existe glucosa disponible (13). También estimula la producción de esteroides en las células ováricas (14).

El sistema IGF (Factor Insulínico de Crecimiento), compuesto por IGF-I, IGF-II, sus receptores y proteínas ligantes, tienen un rol importante en la modulación del nivel nutricional en el desarrollo folicular (15). El IGF-I es producido en el hígado y tiene efectos reguladores en muchos tipos de células. Las concentraciones de IGF-I en sangre disminuyen durante la restricción alimenticia (16). Por lo menos seis proteínas de unión de alta afinidad (IGFBP) pueden influenciar las funciones del IGF-I (17).

Los folículos sintetizan IGF-I y este influye en la función ovárica (14). Específicamente, la proliferación celular ovárica y la esteroidogénesis son estimuladas por IGF-I.
Los folículos dominantes presentan menor actividad IGFBP que los subordinados (18), esta disminución puede incrementar la disponibilidad de IGF-I para las células foliculares. Adicionalmente, las concentraciones de IGFBP-4 parecen determinar cual folículo se vuelve dominante durante la selección (19).

Las concentraciones plasmáticas de estradiol están altamente correlacionadas con los niveles de IGF-I (20). El IGFBP-3 parece ser importante en el transporte de IGF-I hacia tejidos blanco y se ha encontrado en mayor cantidad en plasma de vacas con un folículo ovulatorio durante las tres primeras semanas posparto.

Desde el descubrimiento de la leptina, ha existido mucho interés acerca de su función como señal de información acerca de las reservas energéticas (21). La restricción nutricional incrementa la expresión de receptores de leptina en el núcleo hipotalámico (22). El consumo de nutrientes influye sobre las cantidades de mRNA para leptina en grasa (23) y sobre las concentraciones de leptina en plasma. Las concentraciones de leptina en plasma disminuyen durante el balance energético negativo.

Dentro del sistema nervioso central, el hipotálamo es el sitio de mayor acción de la leptina con respecto al control de consumo de alimento y gasto de energía. Numerosos estudios han evaluado la localización del receptor de RNA mensajero (mRNA) para leptina dentro del hipotálamo (22). El hipotálamo convierte las señales de la leptina en respuestas neuronales sobre el consumo de alimento. El Neuropéptido Y (NPY) parece ser importante en la regulación del consumo. La leptina inhibe las señales del NPY inhibiendo el consumo de alimento (24).

La administración de leptina también estimula la producción de gonadotropinas en la hipófisis por medio de estimulación de $\mathrm{GnRH}$ en el hipotálamo. En rumiantes, la administración de leptina recombinante ovina en vacas de carne maduras sometidas a ayuno estimuló la secreción de LH. En vacas de leche ovariectomizadas afectó la secreción de $\mathrm{LH}$ en relación directa con la dosis aplicada (23). 
En un estudio realizado por Liefers et al (25), no se encontró relación entre los niveles de leptina y la primera actividad luteal posparto, aunque la leptina tiende a incrementarse durante la primera ovulación, indicando la existencia de mecanismos reguladores adicionales en el hipotálamo, hipófisis y ovarios.

Durante la preñez los niveles de leptina son altos y disminuyen rápidamente luego del parto. Esta caída es debida a los costos energéticos de la producción de leche (26). El estimulo de la lactancia por si mismo no parece influenciar la disminución de las concentraciones de leptina (27).

AMAMANTAMI ENTO. El amamantamiento afecta la actividad del hipotálamo, hipófisis y ovarios, mediante la reducción de liberación de $\mathrm{GnRH}$, la cual conduce a insuficientes pulsos de LH (28). Debido a esto los folículos son incapaces de madurar y por lo tanto de ovular, ya que existe una incorrecta síntesis de estrógenos a nivel folicular. Adicionalmente el amamantamiento genera la secreción a nivel hipotalámico de bendorfina, en respuesta al estimulo de succión, y los estrógenos producidos en la placenta durante el último tercio de la gestación provocan la inhabilitación de la secreción de LH a través de la inhibición en el hipotálamo (29).

En el parto, las concentraciones de LH en la hipófisis anterior se agotan debido al efecto inhibitorio de los estrógenos placentarios. El efecto inhibitorio del estradiol acaba con el parto, y al cabo de 2-3 semanas las reservas de LH son similares a las de vacas cíclicas (4). El amamantamiento del ternero prolonga la demora en la aparición de la secreción pulsátil de $\mathrm{LH}$, en vacas que pierden su ternero al nacimiento comúnmente muestran estro mas temprano (5). La administración de antagonistas de opioides incrementa la secreción de LH en vacas lactantes (28). El efecto del amamantamiento es de mayor magnitud en vacas primíparas y en vacas en baja condición (6), las vacas desarrollan un vínculo con su cría, y el efecto del ternero propio es mayor que el de un ternero ajeno.

Estudios iniciales con mastectomia (6) o destete (28), sugieren que el amamantamiento inhibe la reanudación de los pulsos de LH a través de la estimulación táctil, visual y olfativa.

En ganado Costeño con Cuernos (10) se observó, en cuanto al comportamiento de amamantamiento, que las vacas amamantaban a sus crías en determinadas horas del día, mientras que la mayor parte del tiempo permanecían solas alimentandose y las crías permanecían en grupo, con presencia de una hembra nodriza.

\section{OTROS FACTORES}

Efecto del estrés calórico en el eje hipotálamo - hipófisis. Con respecto al patrón de secreción de LH en vacas bajo estrés de calor se han reportado disminuciones en la amplitud (30) y frecuencia (31) de los pulsos. La disminución de los niveles de LH durante el pico preovulatorio ha sido controversial y se ha relacionado con los niveles preovulatorios de estradiol.

La poca información que existe disponible acerca de el efecto del estrés calórico sobre las concentraciones sanguíneas de FSH e inhibina sugieren que la FSH se incrementa y que esto puede ser debido a la disminución en la producción de inhibina (32).

Wilson et al (33) encontraron que el estrés calórico no tuvo efectos sobre la producción de progesterona pero sí retrasó la luteolisis. Otros reportes han sido controversiales (33); estas diferencias probablemente se deban a otros factores no controlados como el tipo de estrés calórico (agudo o crónico) y las diferencias en el consumo de materia seca (32).

Los mecanismos mediante los cuales el estrés calórico afecta las concentraciones de hormonas reproductivas son desconocidos. Se ha sugerido como causa el incremento en la secreción de corticosteroides porque estos pueden inhibir la secreción de GnRH y por lo tanto de LH. La secreción de gonadotropinas es inhibida en mayor grado en vacas con bajas concentraciones plasmáticas de estradiol, sugiriendo que las altas concentraciones de estradiol pueden neutralizar el efecto del estrés calórico (30). 
I nvolución uterina. La involución uterina no está relacionada con la duración del anestro posparto (34), sin embargo, la involución es una barrera física en el posparto temprano tanto para el transporte del esperma como para la implantación (6), muy pocas vacas pueden presentar estro tan temprano como para que la involución uterina interfiera con la concepción, a menos que condiciones patológicas prolonguen la involución normal.

La involución uterina se completa dentro de las 4 a 6 semanas posparto y ocurre más rápidamente en el cuerno no grávido de la preñez anterior (35)

Edad (número de partos). Debido a que las vacas de primer parto continúan en crecimiento, la frecuencia de pulsos de LH en el posparto es menor, y el anestro posparto se prolonga de 1 a 4 semanas más que en vacas multíparas (36).

Raza. En vacas de lechería especializada, los folículos medianos $(5-10 \mathrm{~mm})$ son detectables a partir del día 5 posparto. El primer folículo dominante que se desarrolla logra la ovulación entre los días 15 a 27 posparto en la mayoría de vacas de leche (20).

En vacas de carne lactantes, folículos medianos se encuentran desde los días 57 posparto, y su número y tamaño se incrementa a medida que transcurre el posparto. Los folículos dominantes son detectables en los días 10-21 posparto, sin embargo, la mayoría de estos folículos no llegan a ovular, debido a que sufren atresia antes de obtener el tamaño óptimo de ovulación, posiblemente debido a la sensibilidad del eje hipotálamo-hipofisiario al efecto de feedback negativo del $17 \alpha$ estradiol ovárico que retrasa el restablecimiento de los pulsos de LH (36).

En vacas Bos indicus en el trópico, bajo sistema de manejo extensivo con amamantamiento, la actividad ovárica se ha caracterizado por el crecimiento y regresión de folículos de 4-6 $\mathrm{mm}$ con dominancia esporádica a partir de los 26 días posparto, restableciendo la ciclicidad a los 217-278 días (37).

En ganado criollo Costeño con Cuernos, manejado en pastoreo continuo con amamantamiento en el valle del Sinú, se ha observado estro con ovulación y reanudación de la ciclicidad ovárica a partir del día 51 posparto (10).

Factores sociales. La presencia del macho (6), así como las interacciones entre hembras, la presencia de otras hembras en celo y el encadenamiento de conductas sexuales en grupos de hembras, afectan la duración del intervalo posparto (11).

\section{NÁMI CA FOLICULAR DURANTE EL POSPARTO}

Aunque los papeles de las gonadotropinas y otros reguladores endocrinos de la función folicular durante el postparto temprano no están claros, la ultrasonografía hace posible comparar los cambios en el desarrollo folicular ovárico con variables endocrinas y metabólicas para encontrar el origen endocrino del anestro prolongado (38).

El crecimiento folicular y la habilidad de los folículos para producir cantidades considerables de estradiol dependen de un adecuado aporte de FSH y LH (39). La FSH controla el crecimiento folicular, mientras que la LH está asociada con el mantenimiento de los folículos dominantes y la producción de estradiol necesaria para la inducción del estro, el pico preovulatorio de LH y la ovulación (40). La síntesis de estradiol por las células de la granulosa dependen del suministro de substrato aromatizable (predominantemente androstenediona) de las células de la teca interna adyacentes y gobernadas por LH (41).

La reanudación del crecimiento folicular luego del parto se debe al incremento de la secreción de FSH a los 3-4 días posparto en vacas bien alimentadas (42), pero la habilidad de los folículos para desarrollarse y ser dominantes depende de la secreción pulsátil de $\mathrm{LH}$, que a su vez refleja la secreción de GnRH hipotalámico (7). La falla de los mecanismos que controlan la maduración folicular incluye la inhibición de la liberación pulsátil de mayor frecuencia de LH necesaria para estimular la mayor secreción de estradiol, o la inhibición central de liberación de GnRH en respuesta al estradiol (7).

Los patrones de crecimiento y desarrollo 
folicular son inconsistentes luego del parto. Algunas vacas presentan ovarios relativamente inactivos ( $\sin$ crecimiento folicular mayor a $10 \mathrm{~mm}$ ), mientras otras muestran folículos de diámetro mayor a 10 $\mathrm{mm}$ dentro de los 10 a 15 días después del parto (43). La inactividad ovárica puede deberse a insuficiente secreción de LH asociada con un inadecuado consumo de energía. Este tipo de anestro es común en vacas primíparas en las cuales el balance energético negativo es más evidente (43).

El desarrollo de ondas foliculares ocurre temprano en el posparto tanto en ganado de carne como de leche. Posteriormente al parto existe un incremento dramático en la FSH que es seguido por la emergencia de la primera onda folicular (2-7 días posparto) (44). Sin embargo, la ovulación del folículo dominante solamente ocurre cuando este es expuesto a una adecuada frecuencia de pulsos de LH (aproximadamente 1 pulso/h), que permita el incremento en el tamaño del folículo y la producción de estradiol, la cual eventualmente puede conllevar a un pico de LH y una ovulación (45). Se ha determinado que el folículo dominante de la primera onda folicular ovula en el $42 \%$ de vacas holstein de alta producción (20) y solamente en el $11 \%$ de vacas de carne lactantes (46).

Los patrones de secreción de gonadotropinas en el periodo posparto también difieren entre $B$. taurus y B. indicus. Bajo las mismas condiciones, vacas $B$. taurus presentaron mayores concentraciones plasmáticas de LH que vacas brahman, adicionalmente, una alta proporción de vacas $B$. taurus tienen una secreción pulsátil de LH mayor que vacas B. indicus (47).

Periodos posparto prolongados (mas de 150 días) son característicos de ganado Bos indicus y cruces $B$. taurus/B. indicus en regiones tropicales, originando pérdidas económicas debido al fracaso en alcanzar el intervalo entre partos deseable de 12 meses. Ruiz-Cortes y Olivera-Angel (37) reportan reanudación de la ciclicidad estral de los 217 a los 278 días posparto en vacas B. indicus lactantes mantenidas en praderas naturales en Colombia, resultando en un intervalo entre partos de 17-19 meses; en este mismo estudio se observó que durante los primeros seis meses después del parto muchas de las vacas tuvieron periodos en los cuales los folículos crecían hasta menos de $6 \mathrm{~mm}$ de diámetro. En ganado criollo Costeño con Cuernos se reporta estro con ovulación a partir de los 51 días posparto (10).

\section{CLOS ESTRALES DE CORTA DURACI ÓN}

Los ciclos de corta duración ocurren normalmente tras la primera ovulación espontánea posparto en la mayoría de vacas (48); la liberación prematura de PGF2 $\alpha$ por el útero, como resultado de los efectos de la baja progesterona (49) y estradiol (50) antes de la ovulación en los receptores de oxitocina y progesterona endometriales parecen ser la causa de la temprana regresión del cuerpo lúteo. La progesterona producida durante la fase luteal corta puede ser necesaria para el establecimiento de una función luteal normal en el siguiente ciclo estral $(6,19)$.

Con base en los reportes revisados en este trabajo, en conclusión, es evidente que el periodo de anestro posparto influye notablemente en la productividad de los sistemas ganaderos. Los principales factores que afectan este periodo son la nutrición y el amamantamiento, y otros factores modulan a estos primeros en mayor o menor grado. En el trópico, las altas temperaturas y la humedad ejercen un efecto sobre la fertilidad de dos maneras: actuando directamente sobre el eje hipotálamo-hipófisis, y afectando el apetito y consumo de materia seca, lo cual incide sobre el balance energético del animal. Existen métodos para controlar la baja fertilidad durante el posparto en el trópico, como el manejo de las condiciones ambientales, la suplementación estratégica, el amamantamiento restringido y el uso de biotecnologías. Se sugiere que aunado a esto se debe considerar el uso de especies adaptadas a las condiciones particulares del trópico y que han demostrado una alta fertilidad en el mismo.

En la actualidad, alrededor del mundo se realizan numerosos trabajos relacionados con aspectos específicos de la nutrición y el 
amamantamiento en el posparto, y la tendencia es profundizar en el conocimiento de aspectos celulares y moleculares cada vez más específicos. El aumento en el conocimiento de este tema permitirá disminuir el intervalo entre partos incrementando así la productividad en regiones tropicales.

\section{REFERENCI AS}

1. Kanuya NL, Matiko MK, Nkya R, Bittegeko SBP, Mgasa MN, Reksen O et al. Seasonal changes in nutritional status and reproductive performance of Zebu cows kept under a traditional agro-pastoral system in Tanzania. Trop Anim Health Prod 2006; 38:511-519.

2. Montiel F, Ahuja C, Body condition and suckling as factors influencing the duration of postpartum anestrous in cattle: a review. Anim Reprod Sci 2005; 85: 1-26.

3. Duffy P, Crowe MA, Boland MP, Roche JF. Effect of exogenous LH pulses on the fate of the first dominant follicle in postpartum beef cows nursing calves. J Reprod Fertil 2000; 118:9-17.

4. Nett TM, Cermak D, Braden T, Manns J, Niswender G. Pituitary receptors for GnRH and estradiol and pituitary content of gonadotropins in beef cows. II. Changes during the postpartum period. Domest Anim Endocrinol 1988; 5: 81- 89.

5. Wettemann RP, Lents CA, Ciccioli $\mathrm{NH}$, White, FJ, Rubio I. Nutritionaland suckling-mediated anovulation in beef cows J Anim Sci 2003; 81(Sup 2): E48-E59.

6. Short RE, Bellow RA, Staigmiller RB, Berardinelli J G, Custer EE. Physiological mechanisms controlling anestrous and fertility in postpartum beef cattle. J Anim Sci 1990; 68: 799-816.

7. Jolly PD, McDougall S, Fitzpatrick LA, Macmillan KL, Entwistle KW. Physiological effects of undernutrition on postpartum anoestrus in cows. J Reprod Fert 1995; 49 (Suppl.): 477-492.
8. Canfield RW, Butler WR. Energy balance, first ovulation and the effects of naloxone on $\mathrm{LH}$ secretion in early postpartum dairy cows. J Anim Sci 1991; 69: 740-746.

9. Zurek E, Foxcroft OR, Kennelly JJ. Metabolic status and interval to first ovulation in postpartum dairy cows. J Dairy Sci 1995; 78: 1909-1920.

10. Báez GM, Grajales HA, Pérez JE. Caracterización del ciclo estral mediante perfiles de esteroides (progesterona, 17 â-estradiol) en la raza Costeño con cuernos (Bos taurus) en el trópico Colombiano. Livestock Research for Rural Development 2007; Volume 19, Article \#132. Acceso marzo 31, 2009, disponible en http: //www.cipav. org.co/ Irrd/Irrd19/9/baez19132.htm

11. Galina CS, Arthur GH. Review of cattle reproduction in the tropics. 3. Puerperium. Anim Breed Abstr 1989; 57: 889-910.

12. Villa-Godoy A, Hughes, TL, Emery RS, Chapin LT, Fogwell RL. Association between energy balance and luteal function in lactating dairy cows. J Dairy Sci 1988; 71: 1063-1072.

13. Arias $P$, Rodriguez $M$, Szwarcfarb $B$, Sinay IR, Moguilevsky JA. Effect of insulin on LHRH release by perifused hypothalamic fragments. Neuroendocrinology 1992; 56: 415-418.

14. Spicer LJ , Echternkamp SE. The ovarian insulin and insulin-like growth factor system with an emphasis on domestic animals. Domest Anim Endocrinol 1995; 12: 223-245. 
15. Constant F, Ponter AA, Ponsart C, Fontaine JJ, Mialot JP, Grimard B et al.. Effect of underfeeding on plasmatic and intrafollicular IGFBPs in postpartum anoestrus beef cows. J Reprod Fertil Abstr Ser 2000; 26: 15.

16. Richards MW, Wettemann RP, Spicer LJ, Morgan GL. Nutritional anestrus in beef cows: Effects of body condition and ovariectomy on serum luteinizing hormone and insulin-like growth factorI. Biol Reprod 1991; 44:961-966.

17. Jones JI, Clemmons DR. Insulin-like growth factors and their binding proteins: Biological actions. Endocr Rev 1995; 16:3-34.

18. Yuan W, Bao B, Garverick HA, Youngquist RS, Lucy MC. Follicular dominance in cattle is associated with divergent patterns of ovarian gene expression for insulin-like growth factor (IGF)-I, IGF$\mathrm{II}$, and IGF binding protein-2 in dominant and subordinate follicles. Domest Anim Endocrinol 1998; 15: 55-63.

19. Mihm. M. Delayed resumption of cyclicity in postpartum dairy and beef cows Reprod Domest Anim 1999: 34: 276-284.

20. Butler WR. Energy balance relationships with follicular development, ovulation and fertility in postpartum dairy cows. Livest Prod Sci 2003; 83: 211-218

21. Spicer LJ. Leptin: A possible metabolic signal affecting reproduction. Domest Anim Endocrinol 2001; 21: 251-270.

22. Dyer CJ, Simmons J M, Matteri RL, Keisler $\mathrm{DH}$. Leptin receptor mRNA is expressed in ewe anterior pituitary and adipose tissues and is differentially expressed in hypothalamic regions of well-fed and feed-restricted ewes. Domest Anim Endocrinol 1997; 14:119-128.

23. Amstalden M, Garcia MR, Williams SW, Stanko RL, Nizielski SE, Morrison SD et al. Leptin gene expression, circulating leptin, and luteinizing hormone pulsatility are acutely responsive to short-term fasting in prepubertal heifers: Relationships to circulating insulin and insulin-like growth factor-I. Biol Reprod 2000; 63: 127-133.

24. Jang M, Mistry A, Swick AG, Romsos DR. Leptin rapidly inhibits hypothalamic neuropeptide $Y$ secretion and stimulates corticotropin-releasing hormone secretion in adrenalectomized mice. J Nutr 2000; 130(11): 2813-20.

25. Liefers SC, Veerkamp RF, Te Pas MFW, Chilliard Y, Van der Lende T. Genetics and physiology of leptin in periparturient dairy cows. Domest Anim Endocrinol 2005; 29(1):227-238.

26. Block SS, Butler WR, Ehrhardt RA, Bell AW, Van Amburgh ME, Boisclair YR. Decreased concentration of plasma leptin in periparturient dairy cows is caused by negative energy balance. J Endocrinol 2001; 171(2):339-48.

27. Brogan RS, Mitchell SE, Trayhurn P, Smith MS. Suppression of leptin during lactation: contribution of the suckling stimulus versus milk production. Endocrinology 1999; 140(6): 2621-7.

28. Williams GL, Gazal, OS, Guzman Vega GA, Stanko RL. Mechanisms regulatin suckling-mediated anovulation in the cow. Anim Reprod Sci 1996; 42: 289-297.

29. Day ML. Hormonal induction of estrous cycles in anestrous bos taurus beef cows. Anim Reprod Sci 2004; 8283: 487-494.

30. Gilad E, Meidan R, Berman A, Graber Y, Wolfenson D. Effect of heat stress on tonic and $\mathrm{GnRH}$-induced gonadotrophin secretion in relation to concentration of oestradiol in plasma of cyclic cows. J Reprod Fertil 1993; 99: 315-21.

31. Wise ME, Armstrong DV, Huber JT, Hunter R, Wiersma F. Hormonal alterations in the lactating dairy cow in response to thermal stress. J Dairy Sci 1988; 71:2480-2485. 
32. De Rensis F, Scaramuzzi RJ. Heat stress and seasonal effects on reproduction in the dairy cow - a review. Theriogenology 2003; 60: 1139-1151.

33. Wilson SJ, Marion RS, Spain J N, Spiers DE, Keisler DH, Lucy MC. Effect of controlled heat stress on ovarian function in dairy cattle: I. Lactating cows. J Dairy Sci 1998; 1:2124-2131.

34. Kiracofe GH. Uterine involution: Its role in regulating postpartum intervals. J Anim Sci 1980; 51(Supp 2): 16-28.

35. Spicer LJ, Leung K, Convey EM, Gunther $\mathrm{J}$, Short RE, Tucker HA. Anovulation in postpartum suckled beef cows. I. Associations among size and numbers of ovarian follicles, uterine involution, and hormones in serum and follicular fluid. J Anim Sci 1986; 62: 734-741.

36. Yavas Y, Walton JS. Postpartum aciclicity in suckled beef cows: a review. Theriogenology 2000; 54: 25-55

37. Ruiz-Cortez ZT, Olivera-Angel $M$,. Ovarian follicular dynamics in suckled zebu (Bos indicus) cows monitored by real time ultrasonography. Anim Reprod Sci 1999; 54:211-220.

38. Stagg K, Diskin MG, Sreenan J M, Roche JF. Follicular development in long-term anoestrus suckler beef cows fed two levels of energy postpartum. Anim Reprod Sci 1995; 38:49-61.

39. Driancourt, MA. Follicular dynamics in sheep and cattle. Theriogenology 1991; 35:55- 79 .

40. Fortune JE. Ovarian follicular growth and development in mammals. Biol Reprod 1994; 50:225-232.

41. McNatty, KP, Heath DA, Henderson KM, Lun S, Hurst PR, Ellis LM et al. Some aspects of thecal and granulosa cell function during follicular development in the bovine ovary. J Reprod Fertil 1984; 72:39-53.
42. Lamming GE, Wathes DC, Peters AR. Endocrine patterns in the post-partum cow. J Reprod Fert 1981; 30(Supp): 155- 170.

43. Lucy MC, Savio JD, Badinga L, De La Sota RL, Thatcher WW. Factors That Affect Ovarian Follicular Dynamics in Cattle. J Anim Sci 1992; 70:3615-3626

44. Wiltbank MC, Gumen A, Sartori R, Physiological classification of anovulatory conditions in cattle. Theriogenology 2002; 57:21-52.

45. Roche JF, Crowe MA, Boland MP. Postpartum anestrus in dairy and beef cows. Anim Reprod Sci 1992; 28: 371378.

46. Murphy MG, Boland MP, Roche JF. Pattern of follicular growth and resumption of ovarian activity in postpartum beef suckler cows. J Reprod Fertil 1990; 90(2): 523-533.

47. D'Occhio MJ, Neish A, Broadhurst L. Differences in gonadotrophin secretion post partum between Zebu and European breed cattle. Anim Reprod Sci 1990; 22:311-317.

48. Day ML, Dyer RM, Wilson GW, Pope WF. Influence of estradiol on duration of anestrus and incidence of short estrous cycles in postpartum cows. Domest Anim Endocrinol 1990; 7: 19-25.

49. Zollers WG, Garverick HA, Smith MF, Moffatt RJ, Salfen BE, Youngquist RS. Concentrations of progesterone and oxytocin receptors in endometrium of post partum cows expected to have a short or normal oestrous cycle. J Reprod Fertil 1993; 97: 329-337.

50. Mann GE, Lamming GE. The role of suboptimal preovulatory oestradiol secretion in the aetiology of premature luteolysis during the short oestrous cycle in the cow. Anim Reprod Sci 2000; 64(3-4): 171-80. 